\title{
Echocardiographic evaluation of paravalvular aortic regurgitation of a patient with recurrent aortic valve replacements
}

\author{
Lorenzo Cannata, Camilla Zocco Ramazzo \\ Cardiac Rehabilitation Division, ICS Maugeri IRCCS, Gattico-Veruno (NO), Italy
}

\begin{abstract}
Paravalvular regurgitation (PVR) is a serious complication after surgical valve replacement. Echocardiography is the gold standard technique to assess the severity of PVR with anintegrated approach. A 48-year-old male underwent aortic valve replacement due to infective endocarditis (IE). During in-hospital cardiac rehabilitation after a redo surgery for a new IE three years later, an echo-free anterior space around the valve with several PVRs through transthoracic echocardiography (TTE) was identified. On transesophageal echocardiography (TOE) a double PVR (anterior and posterior) with abscessual cavity was detected. The patient's conditions worsened within six months with hemodynamic instability: a massive PVR due to a large abscess cavity next to the right cusp was confirmed by TTE and TOE and a third surgery was carried out. On the third admission at our
\end{abstract}

Correspondence: Lorenzo Cannata, Cardiac Rehabilitation Division, ICS Maugeri IRCCS, Gattico-Veruno (NO), Italy.

Tel. +39.3756021089

E-mail: lorenzo.cannata@icsmaugeri.it

Key words: Echocardiography; Paravalvular regurgitation; Aortic valve replacement; Infective endocarditis.

Author Contributions: All the authors made a substantive intellectual contribution. All the authors have read and approved the final version of the manuscript and agreed to be accountable for all aspects of the work.

Conflict of interest: The authors declare that they have no competing interests, and all authors confirm accuracy.

Informed Consent: The authors confirm that written consent for submission and publication of this case report including images has been obtained from the patient.

Received for publication: 11 January 2021

Accepted for publication: 12 April 2021.

${ }^{\circ}$ Copyright: the Author(s), 2021

Licensee PAGEPress, Italy

Monaldi Archives for Chest Disease 2021; 91:1765

doi: 10.4081/monaldi.2021.1765

This article is distributed under the terms of the Creative Commons Attribution Noncommercial License (by-nc 4.0) which permits any noncommercial use, distribution, and reproduction in any medium, provided the original author(s) and source are credited.
Rehabilitation Unit the echo features were normal. Patient did not report any cardiovascular symptoms at 1 year follow-up.

\section{Introduction}

Paravalvular regurgitation (PVR) is a serious complication after surgical or transcatheter valvereplacement therapy defined as an abnormal communication between the implanted prosthesis structure and the surrounding cardiac tissue, generating a turbulent blood flow with varied clinical consequences [1-7]. Echocardiography remains the primary imaging modality for assessing aortic PVR, including prosthesis with infective endocarditis (IE). However, the evaluation and grading of PVR is complicated by the complex nature of jets after valve implantation and by the acoustic prosthesis' shadowing. Similar to any assessment of regurgitation, evaluation of PVR should be an integrated approach with the usage of different qualitative parameters as well as semi-quantitative and quantitative measurements [2].

We report the case of a 48-year-old man who underwent recurrent aortic valve replacement due to IE. Echocardiography was adopted for the diagnosis and evaluation of PVR.

\section{Case Report}

The patient, without significant past medical history, underwent transthoracic echocardiogram(TTE) because of sport eligibility in the local medical centre in 2001. The echocardiographic examination showed normal morphological structures of the aortic valve with mild regurgitation. Three years later, the patient presented to the emergency room with shortness ofbreath. On physical examination, the patient appeared well and was alert and interactive. The temperature was $39^{\circ} \mathrm{C}$. The blood pressure was $90 / 50$ $\mathrm{mmHg}$ and the pulse 115 beats per min (bpm). Cardiac examination showed holosystolic murmur. The blood CRP was $18 \mathrm{mg} / \mathrm{L}$ and the leukocytes were $15.2 \times 10^{9} / 1$. TTE and transoesophageal echocardiogram (TOE) showed a large mobile vegetation on the right cusp of the aortic valve with severe regurgitation. The patient was handled with valve replacement in October 2004 and a biological prosthesis (Medtronic n. 27) in order to avoid life-long anticoagulant therapy was chosen. On admission at cardiac rehabilitation centre, the patient was asymptomatic with no cardiac murmurs; lungs' auscultation was normal. Electrocardiogram (ECG), chest X-ray and blood- tests were as expected after cardiac surgery. A TTE showed a left ventricular concentric remodelling (mass index: $94 \mathrm{gr} / \mathrm{m}^{2}$, RWT 0.72) and a well-seated aortic valve 
prosthesis with normal gradients (mean $10 \mathrm{mmHg}$ ) and mild paravalvular regurgitation (PVR) [vena contracta width $0.3 \mathrm{~cm}$, pressure half time (PHT) $525 \mathrm{msec}$. The patient was discharged on oral anticoagulant (Warfarin) and beta-blockers therapy. Subsequent echocardiography follow-up showed progressive degeneration of the prosthesis up to severe stenosis in 2018.

Seven months later, in February 2019, the patient presented to the cardiology outpatient department with dyspnoea, temperature $38.7^{\circ} \mathrm{C}$, blood pressure $120 / 50 \mathrm{mmHg}$ and $95 \mathrm{bpm}$. Chest examination was normal while cardiac examination showed olodiastolic murmur. Bloodcultures were positive for Staphylococcus aureus. Moreover, at both the TTE and TOEexaminations an IE involving bioprosthesis was substantiated. A redo valve replacement was done in March 2019 with bioprosthesis Edwards Inspiris Resilia n. 27, followed by antibiothicterapy (Daptomicin $10 \mathrm{mg} / \mathrm{kg} /$ day) for six weeks.

On admission at rehabilitation centre 9 days after the second replacement, the patient was asymptomatic, apyretic and haemodynamically stable. Oxygen saturation was $96 \%$, while blood pressure was $110 / 70 \mathrm{mmHg}$. There were no cardiac murmurs and the

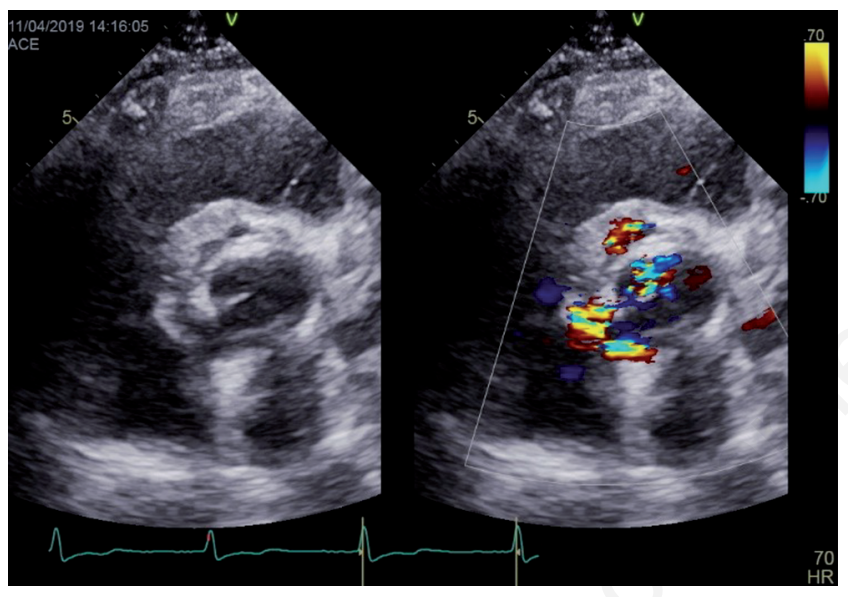

Figure 1. Transthoracic echocardiography parasternal short axis view on aortic bioprosthesis, circumferential extent of jets $50 \%$.

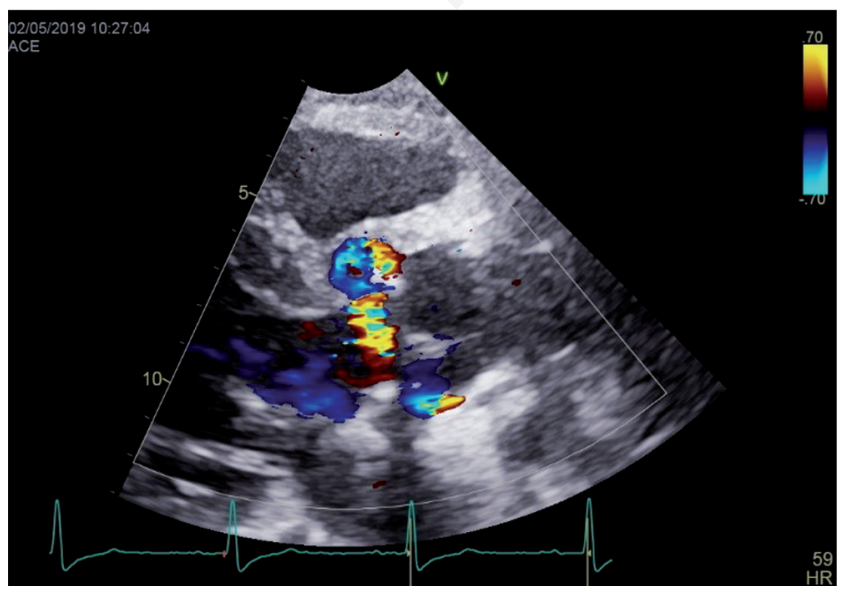

Figure 2. Transthoracic echocardiography parasternal long axis view on aortic bioprosthesis, vena contracta $6 \mathrm{~mm}$. auscultation of the lungs was normal. In addition, ECG Holter showed first degree atrioventricular block. Chest X-ray and blood tests were normal.

TTE showed moderate dilation of the left ventricle, with concentric hypertrophy (mass index: $122 \mathrm{gr} / \mathrm{m}^{2}$, RWT 0.54) and preserved global systolic function (EDVi $93 \mathrm{ml} / \mathrm{m}^{2}$, EF 57\%). The bioprosthesis appeared well-seated with high gradients in line with those provided by the manufactures (mean $21 \mathrm{mmHg}$ ). The aortic root was dilated (diameter $44 \mathrm{~mm}$ ). A mild intraprosthetic central jet was detected. An echo-free anterior space around the valve with multiple paravalvular regurgitation jets was visualized: overall regurgitation was evaluated as severe (PHT $310 \mathrm{~ms}$, vena contracta $6 \mathrm{~mm}$, circumferential extent of jets 50\%) (Figures 1 and 2). In order to better identifying and grading PVR, a TOE was performed and showed severe double PVR (anterior and posterior) and abscessual cavity next to the non-coronary and right-coronary sinus. A communication between these formations was suspected, since evident systolic-diastolic flow inside was present (Figure 3). During hospital stay at rehabilitation unit the patient remained asymptomatic, apyretic and without any clinical signs of infection.

The patient was discharged with beta-blockers, ACEinhibitors and antiplatelet therapy and underwent clinical and echocardiographic follow-up in order to monitor the PVR. Unfortunately, the patient's conditions abruptly worsened. He complained severe dyspnoea.Blood pressure drop to $65 / 35 \mathrm{mmHg}$. In the Cardiothoracic Department, a massive PVR due to a large abscess cavity next to the right cusp was confirmed by TTE and TOE examinations. In October 2019 a mechanical aortic valve replacement (Sorin Bicarbon n. 22) and aortic rootreinforcement were carried out.

The patient was admitted to Cardiac Rehabilitation Unit for the third time one week after surgery. He was, once again, asymptomatic, apyretic, with no cardiac murmurs; the auscultation of the lungs was normal. Furthermore ECG, chest-XR and blood-test were normal. TTE showed normal morpho-functional aspects of the mechanical prosthesis (mean gradient: $10 \mathrm{mmHg}$ ) with a mild washing jet. An ectasic aortic root (44 mm) was confirmed, with the abscessual cavity anatomically and functionally excluded. Other echo features were normal. He was discharged on oral anticoagulant therapy (Warfarin), beta-blockers and ACE-inhibitors. Patientdid not report any cardiovascular symptoms at 1 year follow-up.

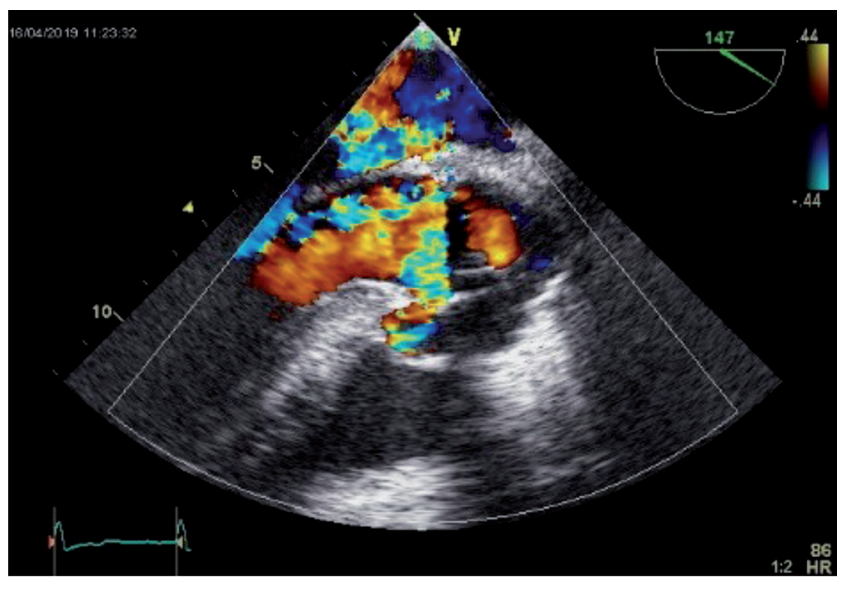

Figure 3. Transesophageal echocardiography on aortic bioprosthesis, abscessual cavity. 


\section{Discussion}

Aortic valve replacement (AVR) is the core of aortic valve IE treatment in the presence of large vegetation or severe valvular degeneration. The choice of prosthesis in AVR for IE patients is controversial since guidelines give no specific recommendations for selection. Prosthetic valve endocarditis (PVE) occurs in 1.6\% of patients with valve prosthesis $(1.25 \%$ patient per-year) $[3,6]$. PARTNER Trial 4 analysed 8530 patients treated with AVR: death from any cause occurred in 3129 patients (46.4\%) over the course of 5 years:107 patients developed PVE and the mortality occurred in 53 patients (49.5\%). Current literature reports high rates of complications (at least 1 complication in $87 \%$ of cases) of PVE, including paravalvular and aortic root abscesses (15.1\%) and intra/paravalvular regurgitation $(15.1 \%)$. In particular, the incidence of PVR post-AVR is $8.6 \%$ in PVE and $6.7 \%$ in nonPVE, respectively [4].

Initial diagnosis of PVR can be a challenge even for an expert cardiologist as clinical presentation depends mainly on the regurgitation's severity. According to current guidelines, the first-line instrumental approach to detect PVR is 2D-TTE due to its availability, wide versatility, radiation-free, low cost and above all diagnosis accuracy. The initial assessment of PVR includes a description of prosthetic valve morphology: sewing ring stability/motion or any anomalous space between the sewing ring and native annulus may be the first evidence of PVR. With ultrasound plane rotation, tilting upward or sideways, a complete evaluation of the prosthesis can be achieved. The implementation of unconventional plans may foreshorten cardiac structures as they are fundamental in detecting the PVR. Indeed, the importance of apical views lies in the fact that some jets may not be localized in the parasternal views because of shadowing from the prosthesis. Subsequently, high-quality Doppler evaluation and a multiparametric/multiwindows assessment are required. The most helpful parameters include AR velocity waveform density (CWD) and diastolic flow reversal (PWD) in proximal descending aorta. Moreover, vena contracta width, jet deceleration time, circumferential extent of PVR, regurgitant volume (RV) and effective regurgitant orifice area (EROA) can be semiquantitative and quantitative supportive measures (Table 1).

In some cases, aortic regurgitation jets can be incorrectly detected by TTE; for this reason, TOE should be performed. TOE is not only essential for the evaluation and treatment strategy, but itis also the key for guidance during the open-heart surgery or percutaneous PVR repair. Particularly, 3D-TOE significantly improves the diagnostic accuracy due to a better spatial resolution and a more detailed quantification of multiples PVR degree. This tool allows to acquire a 3D colour full volume that can then be rotated and cropped for the precise delimitationof the EROA. Besides, feasibility of 3D-TOE is strongly operator-depending since it is conditioned by the mandatory acquisition of high-quality images $[2,5,8,9]$.

As quite recent study analysed TTE/TOE assessment of PVR in valves affected by IE in comparison with intraoperative findings: sensitivity, specificity, positive and negative predictive value were all $100 \%$ [10].

Cardiovascular magnetic resonance (CMR) is an attractive technique that provides precise andreproducible direct quantification of PVR with a measure of RV irrespective of the number of regurgitant jets or their morphology. Nonetheless, a wide clinical application of this technique is limited by high costs and difficult access to scanners: CMR should be considered especially when clinical presentation and TTE/TOE results do not concur $[2,11]$.

In fact, at $\mathrm{CMR}, \mathrm{RV}$ and regurgitant fraction (RF) are significantly ( $p$-value $<0.0005$ ) correlated with PVR severity at TTE. A cut-off value of $\mathrm{RF}<14 \%$ at CMR accurately discriminated mild from moderate/severe (sensitivity: 100\%, specificity: $82 \%$ ) [12].

Furthermore ECG-gated CT and PET are useful modalities in the diagnosis of PVR but also for IE or paravalvular abscess and pseudoaneurysm thanks to their superior spatial and adequate temporal resolutions [10].

These two modalities are required to better visualized and characterized the size, shape and exact location of the PVR defects. Moreover, many studies show a high concordance between ECG-gated CT findings and surgical findings regarding the location and localization of PVR (Figure 4) [13].

In our case report, 2D-TTE/TOE alone provided prompt identification and grading of PVR thanks to a good visualization of left ventricle outflow tract region without intercepting the valve prosthesis and avoiding the limitation of acoustic shadowing. A first line detailed echocardiographic evaluation of the prosthetic valve with a report of any detectable morphologic/ functional alteration as well as clinical variables are essential to guarantee optimal management of patients after recently prosthetic valves replacement.

Table 1. Evaluation of severity of prosthetic aortic regurgitation.

\begin{tabular}{|c|c|c|c|}
\hline & Mild & Moderate & Severe \\
\hline \multicolumn{4}{|l|}{ Quantitative } \\
\hline EROA $\left(\mathrm{cm}^{2}\right)$ & $<0.10$ & $0.10-0.29$ & $\geq 0.30$ \\
\hline Regurgitant volume (mL) & $<30$ & $30-59$ & $>60$ (may be lower in low-flow states) \\
\hline \multicolumn{4}{|l|}{ Semi-quantitative } \\
\hline Vena contracta width $(\mathrm{cm})$ & $<0.3$ & $0.3-0.6$ & $>0.6$ \\
\hline Pressure half time(PHT, ms) & $\begin{array}{c}\text { Variable } \\
\text { Usually }>500\end{array}$ & $\begin{array}{l}\text { Variable } \\
200-500\end{array}$ & $\begin{array}{c}\text { Steep } \\
\text { Usually }<200\end{array}$ \\
\hline Circumferential extent of PVR (\%) & $<10$ & $10-29$ & $\geq 30$ \\
\hline \multicolumn{4}{|l|}{ Qualitative } \\
\hline AR wave form density (with CW Doppler) & Subdued & Solid & Solid \\
\hline $\begin{array}{l}\text { Diastolic flow reversal (with PW Doppler) } \\
\text { in proximal descendingaorta }\end{array}$ & Brief, early diastolic & May be holodiastolic & $\begin{array}{c}\text { Holodiastolic } \\
\text { (end- diastolic velocity } \geq 20 \mathrm{~cm} / \mathrm{s} \text { ) }\end{array}$ \\
\hline
\end{tabular}




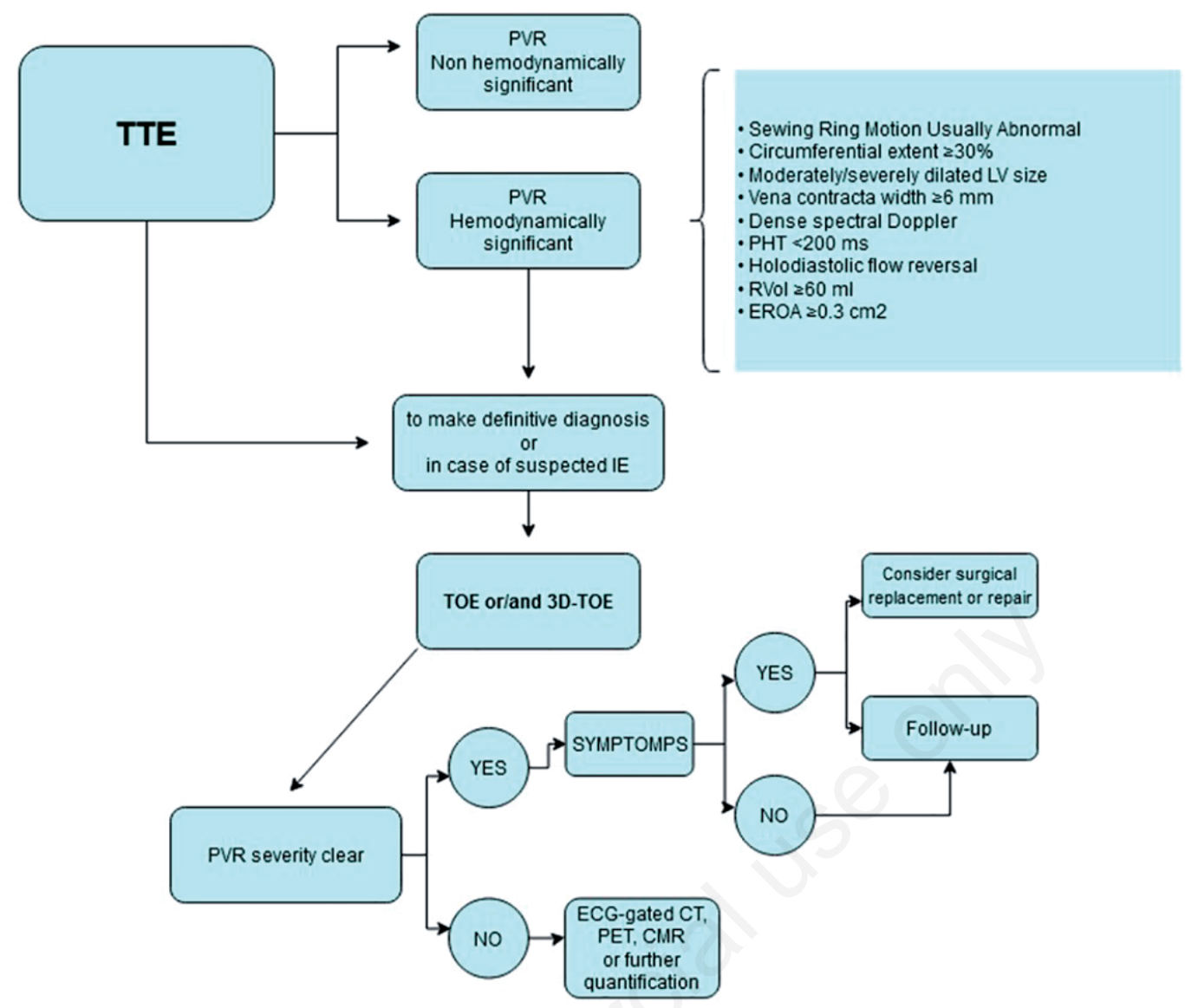

Figure 4. Diagnostic algorithm.

\section{References}

1. Safi AM, Kwan T. Paravalvular regurgitation: a rare complication following valve replacement surgery. Angiology 2000;51:479-87.

2. Zoghbi WA, Asch FM, Bruce C, et al. Guidelines for the Evaluation of Valvular Regurgitation After Percutaneous Valve Repair or Replacement A Report from the American Society of Echocardiography Developed in Collaboration with the Society for Cardiovascular Angiography and Interventions, Japanese Society of Echocardiography, and Society for Cardiovascular Magnetic Resonance. Erratum in: [No authors listed] J Am Soc Echocardiogr 2019;32:914-7. J Am Soc Echocardiog 2019;32:431-75.

3. Habib G, Lancellotti P, Antunes MJ, et al. 2015 ESC Guidelines for the management of infective endocarditis - The Task Force for the Management of Infective Endocarditis of the European Society of Cardiology (ESC). Eur Heart J 2015;36:3075-23.

4. Summers MR, Leon MB, Smith CR, et al. Prosthetic Valve Endocarditis After TAVR and SAVR - Insights From the PARTNER Trials. Circulation 2019;140:1984-94.

5. Ruiz CR, Hahn RT, Alain Berrebi A, et al. Clinical trial principles and endpoint definitions for paravalvular leaks in surgical prosthesis. An expert statement. J Am Coll Cardiol 2017;69:2067-87.

6. Kytö V, Ahtela E, J Sipilä J, et al. Mechanical versus biological valve prosthesis for surgical aortic valve replacement in patients with infective endocarditis. Interact Cardiovasc Thoracic Surg 2019;29:386-92.

7. Pinheiro CP, Rezek D, Costa EP, et al. Paravalvular regurgitation: Clinical outcomes in surgical and percutaneous treatments. Arq Bras Cardiol 2016;107:55-62.

8. Del Val Martìn D, Zamorano Gòmez JL. The role of imaging in paravalvular leak assessment. In: G Smolka, W Wojakowski, M Tendera, Editors. Transcatheter paravalvular leak closure. Springer, Singapore; 2017. p. 13-42.

9. Geleijnse ML, Di Martino LFM, Vletter WB, et al. Limitations and difficulties of echocardiographic short-axis assessment of paravalvular leakage after corevalve transcatheter aortic valve implantation. J Cardiovascular Ultrasound 2016;14:37.

10. Hryniewiecki T, Zatorska K, Abramczuk E, et al. The usefulness of cardiac CT in the diagnosis of perivalvular complications in patients with infective endocarditis. Eur Radiology 2019;29:4368-76.

11. Eleid MF, Cabalka AK, Malouf JF, et al. Techniques and outcomes for the treatment of paravalvular leak. Circ Cardiovasc Interv 2015;8:e001945.

12. Salaun E, Jacquier A, Theron A, et al. Value of CMR in quantification of paravalvular aortic regurgitation after TAVI. Eur Heart J Cardiovasc Imaging 2016;17:41-50.

13. Ghersin E, Martinez CA, Singh V, et al. ECG-Gated MDCT after aortic and mitral valve surgery. Am J Roentgenol 2014;203:W596-604. 